\title{
Article \\ Airspace Capacity Assessment with Augmented Cell Transmission Model
}

\author{
Wei Gao ${ }^{1, \ddagger}$, Man Liang $2, \ddagger(1) *$ \\ 1 Civil Aviation University of China, Tianjin, China \\ RMIT University, Melbourne, Australia; man.liang@rmit.edu.au \\ * Correspondence: man.liang@rmit.edu.au \\ $\ddagger \quad$ These authors contributed equally to this work.
}

\begin{abstract}
Air traffic congestion is caused by the unbalance between increasing traffic demand and saturating capacity. Flight delay not only causes huge economical lost, but also has very negative environmental impact in the whole air transportation system. In order to identify the impact of extended TMA on airport capacity, an airspace capacity assessment method based on augmented cell transmission model was proposed. Firstly, the airspace structure was modeled with points, segments, layers, and cells. Secondly, mixed integer linear programming model was built up with maximum throughput or capacity as the objective function. Finally, genetic algorithm was used to find the optimal result, and the results were validated by comparing with the fast-time simulation results generated by total airspace and airport modeler (TAAM) software. It is found that the proposed method could achieve a relatively accurate result in a much affordable and fast way. The numerical results could be very helpful for air traffic controllers to analyze the dynamic traffic flow entering and exiting TMA, so as to make decisions via reasonable analysis and do planning in advance by referring to the airport capacity.
\end{abstract}

Keywords: Air transportation; air traffic control; airspace capacity; cell transmission model

\section{Introduction}

Air traffic congestion is caused by the unbalance between increasing traffic demand and saturating capacity. Flight delay not only causes huge economical lost, but also has very negative environmental impact in the whole air transportation system. Air delays will normally trigger a large number of aircraft to either hold in the holding stacks for more than 30 minutes, or force aircraft to reroute and fly a longer distance, which require more fuel consumption, consequently causing more pollution in the air.

In the last 30 years, civil aviation in China has been in a fastest development in the world. With the continuous improvements of airspace planning and management, the operational efficiency of Terminal Control Areas (TMAs) at China's busiest airports, such as Beijing, Shanghai and Xi'an, has been largely improved. Thus, the bottleneck of capacity at the airport has been gradually shifting to the extended TMAs, where aircraft are entering or exiting the en-route cruising phase between airways and busy airports.

In order to reduce the delays in extended TMAs, four key factors needs to be considered: flow management, topological structure, traffic complexity, and dangerous weather. Insufficient route capacity will cause heavy traffic delay [1]. Operational cost to mitigate the traffic delay could be changed with dynamic flow management strategies [2]. Thus, an efficient flow management should be able to optimize traffic flow with reference to the different levels of delay, safety cost and capacity. A safety-capacity trade-off approach could be considered to calculate a maximum sector capacity under an acceptable level of safety risk, so as to reduce delay in a practical way [3]. Route network design has naturally impact on capacity and complexity. On one side, the airway converging points layout plays an important role in the network complexity analysis. Normally, the closer and denser the 


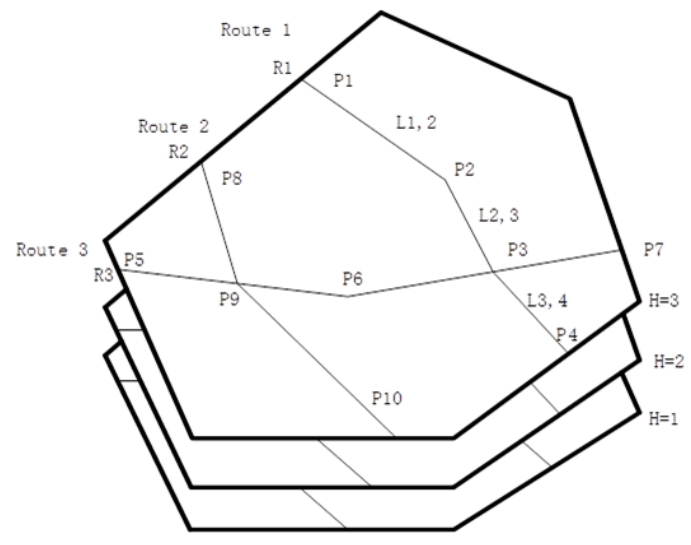

Figure 1. Example of a 3D route network

converging points are in a specific airspace, the route network complexity is getting higher. The complex network modeling is considered to optimize the route convergence point layout problem [4]. On the other side, the number and locations of airway intersections, the length of airway segments, and the angle of airway intersections are also important factors to airway network complexity. By merging and changing some positions of intersections could result in a better route network structure [5]. In addition, other factors, such as traffic flow complexity or traffic pattern, and dangerous weather, have extremely severe impact on route network dynamic performance. Prediction plays crucial role in handling the abnormal situation in airspace, so as to keep the airspace capacity in a reasonable level. By considering the coming traffic flows in 30 minutes' to a few hours' time window, the airspace dynamic capacity could be predicted, then different controllers' strategy and decisions could be made to avoid heavy delays happening in extended TMAs [6]. In term of dangerous weather, prediction normally be made at least hours in advance, so as to avoid chaos happening in extended TMA airspace [7].

The concept of cell transmission model (CTM) was firstly proposed by C. F. Daganzo in 1994, which is a discrete separation form of the kinematic model Lighthill, Whitham and Richards (LWR) [8-10]. This method simulates the dynamic flow in the network by discrete separation and decentralization, which is suitable for the research of macroscopic traffic flow. Through the continuous improvement and perfection of scholars, it has been widely used in the field of road traffic, such as dynamic traffic assignment [11], autonomous vehicle flow control [12]. In the aviation field, some researchers applied CTM to en-route air traffic flow management [13]. However, most of their studies were based on two-dimensional space, which is not the best assumption for aviation. In this paper, we are developing a 3D augmented cell transmission model by adding the altitude to the $2 \mathrm{D}$ model.

\section{Augmented Cell Transmission model}

\subsection{Airspace and cell structure}

As shown in Figure 1, the extended TMA airspace is a three-dimensional space, which could be divided into several different layers with a vertical separation of $1000 \mathrm{ft}$ or $300 \mathrm{~m}$. Each layer is represented by $H_{m}$, as shown in Figure 1 , there are three layers $H_{1}, H_{2}, H_{3}$. On each layer, the two-dimensional route network consists of points, segments, routes, sector boundaries, and cells.

Points $P_{i}, i \in \mathcal{N}$ could be the turning point, the intersection of two different routes, or the crossing point of boundary and route. As shown in Figure 1, there are 10 points, where $P_{2}$ and $P_{6}$ are turning points, $P_{3}$ and $P_{9}$ are route intersections, the others represent different intersections of airspace sector boundary and routes.

A segment is the part between two points $P_{i}, P_{j}$ on the same route, which is represented by $L_{i, j}, i, j \in \mathcal{N}$. For instance, there are 9 segments in Figure 1 , where $L_{1,2}$ represents the 


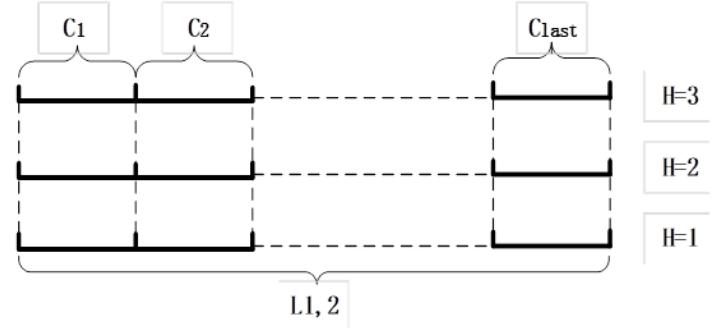

Figure 2. Example of a cell structure in a segment

part of the route between point $P_{1}$ and point $P_{2}$, the direction of which is from point $P_{1}$ to point $P_{2}$, and the direction of the segment $L_{1,2}$ is the same as that of the route.

Routes are composed of segments, which are represented by $R_{k}, k \in \mathcal{N}$. In this paper, routes are divided into two types: 1) routes for aircraft to enter/leave the TMA from/to the extended TMA and 2) routes for aircraft to cruise through the extended TMA airspace. There are three routes in Figure 1. Taking route $R_{1}$ as an example, route $R_{1}$ is composed of segments $L_{1,2}, L_{2,3}, L_{3,4}$. Thus, $R_{1}=\left\{L_{1,2}, L_{2,3}, L_{3,4}\right\}$ and its direction is $P_{1} \rightarrow P_{2} \rightarrow P_{3} \rightarrow P_{4}$. The boundary of a specific airspace is represented by a thick solid line in Figure 1.

Each segment is further divided into several cells according to the control separation minimum, represented by $C_{z}, z \in \mathcal{N}$. For example, in radar control environment, the minimum horizontal separation between two aircraft on the same route in the same direction generally is $5 \mathrm{NM}$. Therefore, each cell could be 5NM wide. Depending on the traffic delay situation, the size of cell is not a fixed value. For instance, if there is a heavy delay in TMA, then the size of cell could be bigger up to 10-15 NM. From an operational point of view, cell is the most basic unit of route network, which demonstrates the spacing between two successive aircraft. It also represents the traffic flow density in a dynamic route network. Taking the segment $L_{1,2}$ as an example, as shown in Figure 2, there are three layers, $H_{1}, H_{2}, H_{3}$, on each layer, there are several cells $C_{1}, C_{2}, \ldots C_{\text {last }}$, and the length of each cell is fixed at time range according to the area control minimum separation or air traffic flow control requirement. Remark that the segment length and the area control separation are not integer corresponding, thus it is necessary to round up the last cell of each segment.

Assume that the length of the cell is $D_{C_{z}}$, the length of the segment $L_{i, j}$ is $D_{L_{i, j}}$, then the total number of cells $n$ in a segment in $L_{i, j}$ is calculated by the following formula:

$$
n=\frac{D_{L_{i, j}}}{D_{C_{z}}}, i, j \in \mathcal{N}
$$

\subsection{Aircraft speed analysis}

By radar data analysis, aircraft ground speed changes with different altitude and position. Figure 3 shows the distributions of aircraft ground speed at two different altitudes, i.e. $31100 \mathrm{ft}$ and $37000 \mathrm{ft}$, in the same segment. Obviously, the mean value and variance of aircraft speed are different at different altitudes. The higher aircraft fly, the faster they travel. As shown in Figure 4, the distributions of aircraft ground speed in different segments at the same altitude is also different. In this case, the mean value of aircraft speed in segment 2 is faster than that in segment 1 . Through data analysis, it is found that majority of aircraft fly within a certain speed range at a specific altitude of a specific segment. Therefore, in this paper, to simplify the study, the mean value of the normal distribution of aircraft speed could be taken as a reference speed, which will not cause excessive calculation error and meet the accuracy requirements. In other words, the reference speed will be the function of altitude $H_{m}$ and cell $C_{z}$. 


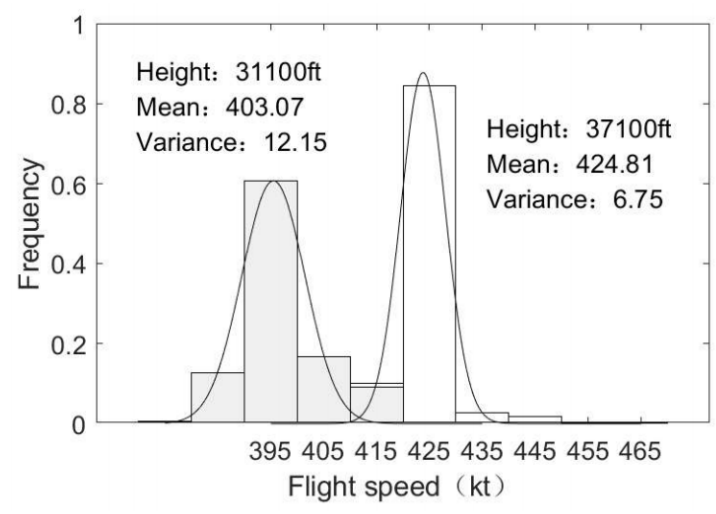

Figure 3. Example of speed changing with altitudes

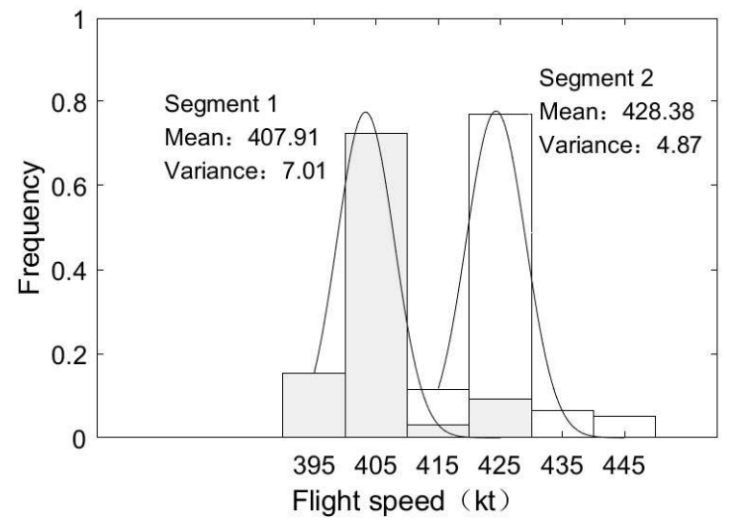

Figure 4. Example of speed changing with segments

\subsection{Cell transmission modelling}

First, several assumptions need to be set up to make a reasonable modelling in a given condition. They are:

- The aircraft ground speed in a cell is constant, and the reference speed is determined by the position and altitude of the segment where the cell is located.

- The time is calculated in minutes.

- The model does not consider the influence of random factors such as weather.

- Cruising aircraft on the overflight route will not change their altitudes.

- When aircraft need to change altitude, it starts from the beginning of one cell and finishes at the end of another cell where this aircraft is going to level off. The total altitude change has to be accomplished within one length of a cell, as shown in Figure 5. The speed of aircraft during level change is the average value of two reference speeds in those two cells.

- Aircraft must not change altitude at all intersections due to safety.

Second, several variables need to be calculated in the cell transmission modelling to describe the aircraft dynamic status. They are: status of aircraft into extended TMA, aircraft

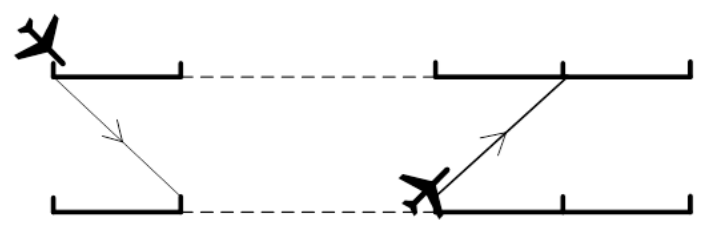

Figure 5. Example of aircraft level change 
level changes, elapsing time inside one cell, entering cell timestamp, exiting cell timestamp. If at absolute time $T_{f}^{E}$, one aircraft $f$ is entering extended TMA from route $R_{k}$ at flight level $H_{m}$, then its status is described by:

$$
f_{R_{k}}^{H_{m}}\left(T_{f}^{E}\right)= \begin{cases}1, & \text { if entering extended TMA } \\ 0, & \text { otherwise }\end{cases}
$$

Assume that aircraft $f$ is entering the extended TMA, then the elapsing time $t$ inside cell $C_{z}, z \in \mathcal{N}$ at flight level $H_{m}$ is described by:

$$
t_{f}\left(C_{z}, H_{m}\right)=\frac{D_{C_{z}}}{v_{f}\left(C_{z}, H_{m}\right)}
$$

where, $v_{f}\left(C_{z}, H_{m}\right)$ is the reference speed of aircraft $f$ inside $C_{z}$ at altitude $H_{m}$.

Along one route $R_{k}$, aircraft altitudes in different cells $C_{z}$ could be described as a matrix:

$$
\mathbf{H}_{\mathbf{f}}^{\mathbf{R}_{\mathbf{k}}}=\left[\begin{array}{cccc}
H_{1}^{C_{1}} & H_{1}^{C_{2}} & \ldots & H_{1}^{C_{\mathcal{Z}}} \\
H_{2}^{C_{1}} & H_{2}^{C_{2}} & \ldots & H_{2}^{C_{\mathcal{Z}}} \\
\vdots & \vdots & \vdots & \vdots \\
H_{m}^{C_{1}} & H_{m}^{C_{2}} & \ldots & H_{m}^{C_{\mathcal{Z}}}
\end{array}\right]
$$

where, $H_{m}^{C_{z}}$ equal to 0 or 1 , and $z \in \mathcal{Z}$. If it is 1 , it means aircraft pass thought $C_{z}$ at altitude $H_{m}$. As mentioned above, the timestamp of aircraft entering the cell depends on the location of the cell $C_{z}$ and the absolute timestamp of entering extended TMA $T_{f}^{E}$. Therefore, the timestamp of entering cell $T_{C_{z}}^{\text {in }}$ could be described by:

$$
T_{C_{z}}^{i n}=\sum_{y=1}^{y=m} f_{R_{k}}^{H_{y}}\left(T_{f}^{E}\right) \times T_{f}^{E}+\sum_{x=1}^{x=z-1} \sum_{y=1}^{y=m} \mathbf{H}_{\mathbf{f}}^{\mathbf{R}_{\mathbf{k}}} \times \mathbf{t}_{\mathbf{f}}\left(\mathbf{C}_{\mathbf{x}}, \mathbf{H}_{\mathbf{y}}\right)
$$

The timestamp of exiting cell $T_{C_{z}}^{o u t}$ will be:

$$
T_{C_{z}}^{o u t}=\sum_{y=1}^{y=m} f_{R_{k}}^{H_{y}}\left(T_{f}^{E}\right) \times T_{f}^{E}+\sum_{x=1}^{x=z} \sum_{y=1}^{y=m} \mathbf{H}_{\mathbf{f}}^{\mathbf{R}_{\mathbf{k}}} \times \mathbf{t}_{\mathbf{f}}\left(\mathbf{C}_{\mathbf{x}}, \mathbf{H}_{\mathbf{y}}\right)
$$

Third, throughput defines the number of aircraft passing in a specific airspace in a specific time range. It is important for calculating the delays. The smallest airspace unit is cell, so the cell throughput could be labelled as $X_{C_{z}}^{R_{k}}$. Then, a sector consists of several routes, and sector throughput is defined as the number of aircraft passing through the sector within a given time, labelled by $S$,

$$
S=\sum_{x=1}^{x=\mathcal{Z}} \sum_{y=1}^{y=\mathcal{M}} X_{C_{x}}^{R_{y}}
$$

Control area includes several sectors, similarly control area throughput, labelled by $A$, could be defined as:

$$
A=\sum_{q=1}^{q=\mathcal{Q}} S_{q}
$$

Fourth, the maximum throughput in a specific airspace is the airspace capacity. By definition, the cell capacity is 1 , which means only 1 aircraft could be flying in one cell due to safety. The objective function is to find the capacity of control area with the hard constraint of only one aircraft in each cell. 


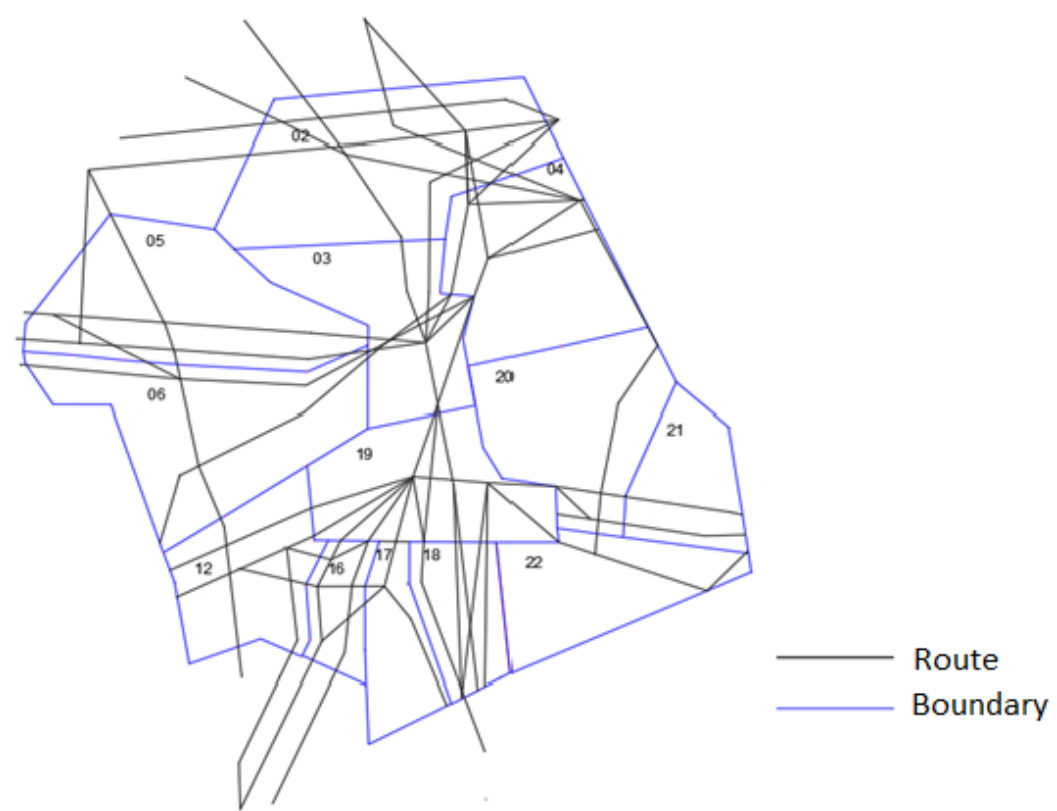

Figure 6. Study case: Beijing extended TMA

\section{Simulation and results}

The study case is the extended TMA around Beijing Capital international airport, with 13 sectors shown in Figure 6. Beijing Capital International Airport was the world's second busiest airport for passenger traffic in pre-COVID period, with 100 million passengers in 2019.

By applying our method, there are 143 way-points, 269 segments, 725 cells and 153 routes. Among the 153 routes, 90 routes pass through the airspace for cruising purpose, 32 routes guide aircraft to descend and enter the TMA, and 31 routes guide the aircraft to climb and enter the extended TMA in area control airspace. The airspace altitude range in Beijing extended TMA is from $7800 \mathrm{~m}$ to $12500 \mathrm{~m}$. According to the cruising level allocation regulations, the routes to the East are divided into 8 levels, i.e. $8100 \mathrm{~m}, 8900 \mathrm{~m}, 9500 \mathrm{~m}$, $10100 \mathrm{~m}, 10700 \mathrm{~m}, 11300 \mathrm{~m}, 11900 \mathrm{~m}$ and $12500 \mathrm{~m}$. While the routes to the West are divided into 8 altitudes, i.e. $7800 \mathrm{~m}, 8400 \mathrm{~m}, 9200 \mathrm{~m}, 9800 \mathrm{~m}, 10400 \mathrm{~m}, 11000 \mathrm{~m}, 11600 \mathrm{~m}$ and $12200 \mathrm{~m}$.

Then Genetic Algorithm (GA) is applied to find the optimal result. GA was first proposed by Professor Holland.J of the University of Michigan in 1975. It is an intelligent search algorithm inspired by the theory of biological evolution [14]. The algorithm uses biological populations to eliminate disadvantaged individuals and retain dominant individuals so that species continue to evolve as a search mechanism to perform multiple iterations of feasible solutions to the problem to select the optimal solution. This method is suitable for complex multi-variables linear programming problems. Compared with traditional algorithms, GA needs to randomly generate a set of initial feasible solutions, and performs iterative selection on the basis of the initial feasible solutions. This initial solution set is called population. Each feasible solution in the solution set is called an individual, and each individual is composed of a string of gene sequences, where the gene sequence is the variable value corresponding to the problem. GA continuously integrates and reorganizes individuals through the selection, crossover, and mutation of gene sequences between individuals to obtain the optimal individual, which is the optimal solution. The flowchart of application of GA in our research is shown in Figure 7.

In the experiment, 1 hour is selected as the total time to calculate the maximum capacity of regional airspace in one hour. Pre-defined parameters are as following:

- the control separation is $20 \mathrm{~km}$,

- the number of individuals in the initial population is 100 ,

- the crossover probability is 0.6 , 


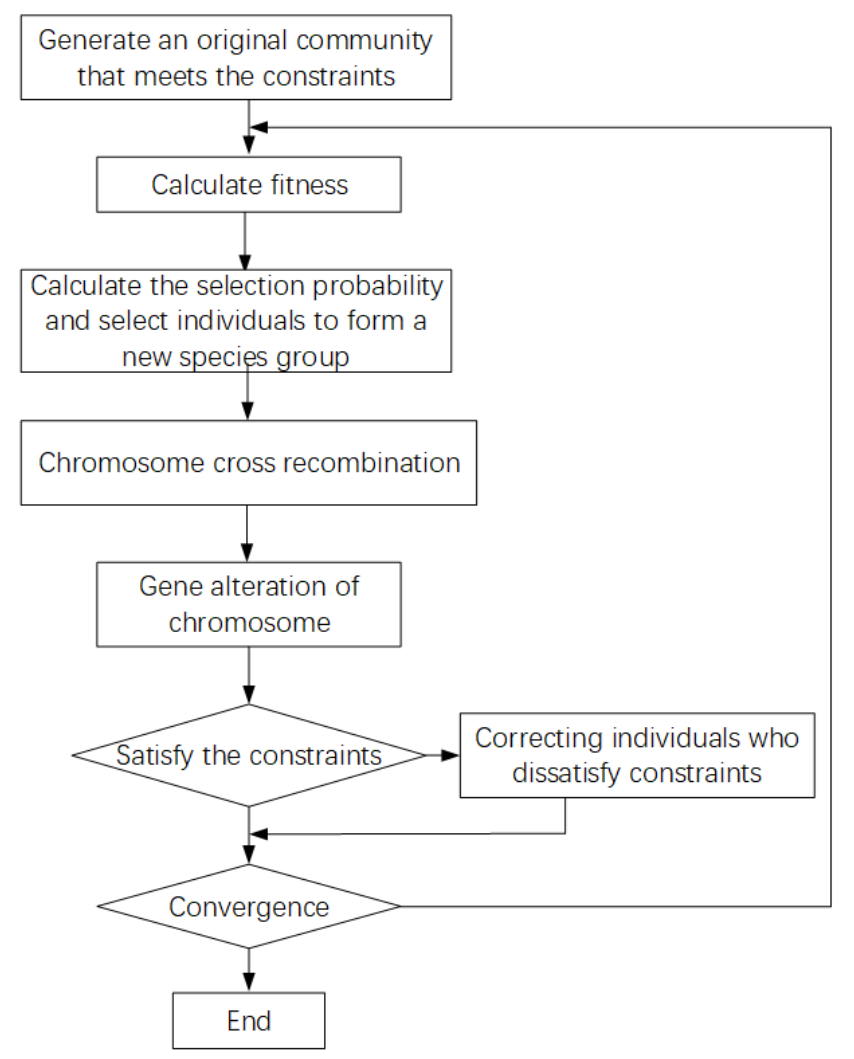

Figure 7. Flowchart of GA method

- $\quad$ the variation probability is 0.01 .

After 60 iterations, the GA results are converging, as shown in Figure 8. The average value of the final convergence is 987.42 . It means the hourly capacity of the airspace is 987 aircraft, of which 185 is the hourly capacity for aircraft entering or leaving the extended TMA from/to TMA, 802 is the hourly capacity for aircraft passing or cruising through the extended TMA.

In order to further validate the Augmented Cell Transmission Model (ACTM), TAAM simulation is applied and compared. TAAM is a fast-time delay and travel time computer simulation model that is being used to calculate delays and travel times. It has been successfully used in many simulation-based capacity assessment, such as runway capacity [15], airspace demand [16]. In this research, an individual of the convergent group from ACTM is selected as a sample to generate a timetable for TAAM. The TAAM simulation results are then compared with those of ACTM model. Table 1 shows the hourly capacity of each sector through different methods, one is ACTM which is proposed in this paper,

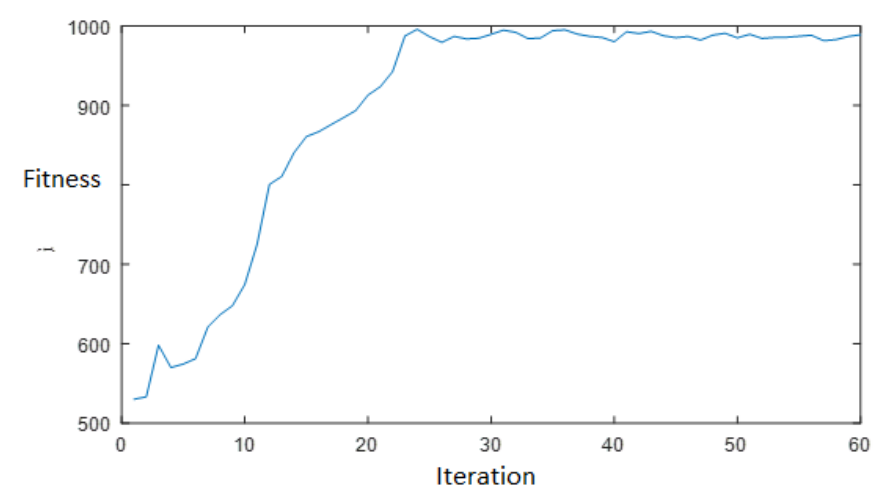

Figure 8. Fitness iteration 
and the other is TAAM. It is found that the traffic flow is mainly concentrated in sector 02 , sector 16, sector 17 and sector 19. Generally, the capacity generated by ACTM is slightly higher than the TAAM simulation results.

Table 1. Results of Hourly Sector Capacity

\begin{tabular}{lll}
\hline Sector ID & Capacity by ACTM & Capacity by TAAM \\
\hline Total airspace & 986 & 975 \\
AR02 & 132 & 128 \\
AR03 & 109 & 103 \\
AR04 & 59 & 53 \\
AR05 & 98 & 94 \\
AR06 & 49 & 47 \\
AR12 & 114 & 109 \\
AR16 & 123 & 121 \\
AR17 & 121 & 119 \\
AR18 & 49 & 45 \\
AR19 & 135 & 131 \\
AR20 & 101 & 98 \\
AR21 & 39 & 39 \\
AR22 & 80 & 78 \\
\hline
\end{tabular}

\section{Conclusions}

In this paper, the augmented 3 dimension cell transmission model is firstly proposed to describe the traffic flow in extended TMA, then genetic algorithm is applied to generate the maximum airspace capacity. The numerical results are validated by comparing with fast-time simulation by TAAM software. This research shows that the proposal ACTM is suitable for the large scale airspace capacity assessment, and does not need to purchase expensive simulation platform, such as TAAM. The modelling method and results are very reliable. As long as the airspace structure is given, its capacity can be analyzed in a fast and affordable way, which is very helpful for air traffic controllers to analyze the dynamic traffic flow entering and exiting TMA, so as to make decisions via reasonable analysis and do planning by referring to the airport capacity. However, in current study, we did not consider the impact from military activities and dangerous weather, which is interesting to discover in the future research.

Author Contributions: Conceptualization, Wei Gao and Man Liang; methodology, Wei Gao and Man Liang; software, Wei Gao ; validation, Wei Gao; writing—original draft preparation, Man Liang; writing-review and editing, Man Liang. All authors have read and agreed to the published version of the manuscript.

Conflicts of Interest: The authors declare no conflict of interest.

\section{References}

1. Bayen, A.M. Computational control of networks of dynamical systems: Application to the national airspace system; stanford university, 2004.

2. Chen, D.; Hu, M.; Zhang, H.; Yin, J.; Han, K. A network based dynamic air traffic flow model for en route airspace system traffic flow optimization. Transportation Research Part E: Logistics and Transportation Review 2017, 106, 1-19.

3. Hossain, M.M.; Sameer, A.; Delahaye, D. An evolutionary computational framework for capacity-safety trade-off in an air transportation network. Chinese Journal of Aeronautics 2019, 32, 999-1010.

4. Cailong, C. Optimization of converging points in route network via complexity network theory. University of Science and Technology of China 2011.

5. Shijin, W.; Xi, C.; Haiyun, L.; Qingyun, L.; Xu, H.; Yanjun, W. Air route network optimization in fragmented airspace based on cellular automata. Chinese Journal of Aeronautics 2017, 30, 1184-1195. 
6. Song, L.; Wanke, C.; Greenbaum, D.; Callner, D. Predicting sector capacity under severe weather impact for traffic flow management. 7th AIAA ATIO Conf, 2nd CEIAT Int'l Conf on Innov and Integr in Aero Sciences, 17th LTA Systems Tech Conf; followed by 2nd TEOS Forum, 2007, p. 7887.

7. Mitchell, J.; Polishchuk, V.; Krozel, J. Airspace throughput analysis considering stochastic weather. AIAA Guidance, Navigation, and Control conference and exhibit, 2006, p. 6770.

8. Daganzo, C.F. The cell transmission model: A dynamic representation of highway traffic consistent with the hydrodynamic theory. Transportation Research Part B: Methodological 1994, 28, 269-287.

9. Lighthill, M.J.; Whitham, G.B. On kinematic waves II. A theory of traffic flow on long crowded roads. Proceedings of the Royal Society of London. Series A. Mathematical and Physical Sciences 1955, 229, 317-345.

10. Daganzo, C.F. The cell transmission model, part II: network traffic. Transportation Research Part B: Methodological 1995, 29 , 79-93.

11. Wang, Y.; Szeto, W.Y.; Han, K.; Friesz, T.L. Dynamic traffic assignment: A review of the methodological advances for environmentally sustainable road transportation applications. Transportation Research Part B: Methodological 2018, 111, 370-394.

12. Levin, M.W.; Boyles, S.D. A multiclass cell transmission model for shared human and autonomous vehicle roads. Transportation Research Part C: Emerging Technologies 2016, 62, 103-116.

13. Sun, D.; Bayen, A.M. Multicommodity Eulerian-Lagrangian large-capacity cell transmission model for en route traffic. Journal of guidance, control, and dynamics 2008, 31, 616-628.

14. Holland, J.H.; others. Adaptation in natural and artificial systems: an introductory analysis with applications to biology, control, and artificial intelligence; MIT press, 1992.

15. Bazargan, M.; Fleming, K.; Subramanian, P. A simulation study to investigate runway capacity using TAAM. Proceedings of the Winter Simulation Conference. IEEE, 2002, Vol. 2, pp. 1235-1243.

16. Rosenow, J.; Fricke, H. Impact of multi-criteria optimized trajectories on European airline efficiency, safety and airspace demand. Journal of Air Transport Management 2019, 78, 133-143. 